\title{
Impact de la télémédecine sur la régulation d'un malaise
}

\author{
Impact of Telemedicine on the Emergency Medical Dispatch of Syncope
}

\author{
M. Dubocage $\cdot$ H. Delelis-Fanien $\cdot$ J. Guenezan
}

Reçu le 16 mai 2017; accepté le 20 décembre 2017

(C) SFMU et Lavoisier SAS 2018

\section{Introduction}

La médecine a toujours bénéficié des progrès de la science, en introduisant dans nos pratiques l'amélioration des outils du quotidien. À l'heure du progrès des télécommunications, il faut envisager son intégration dans le monde de la santé. La télémédecine doit permettre de compléter un arsenal diagnostique parfois limité par la réduction des moyens médicaux. Elle s'intègre pleinement dans l'environnement des patients qui est maintenant devenu de plus en plus numérique.

Nous rapportons le cas d'un patient ayant présenté un malaise avec une douleur thoracique jugée atypique avec intervention d'ambulanciers équipés d'un système de télémédecine (Nomadeec ${ }^{\circledR}$, Exelus, Latresne, France). Il s'agit d'un dispositif avec une ceinture permettant de réaliser un électrocardiogramme (ECG), limitant les risques d'erreurs, notamment dans la pose des électrodes (Fig. 1). Les ECG sont ensuite télétransmis de manière sécurisée par un logiciel spécifique disponible sur les ordinateurs de la régulation du Centre 15.

\section{Observation}

Nous rapportons le cas d'un patient âgé de 70 ans, sans antécédent médical personnel ni facteur de risque cardiovasculaire. Il ne prend pas de traitement et n'a pas d'allergie connue. Il réside dans une zone rurale isolée à $35 \mathrm{~min}$ du centre hospitalier ou du SMUR le plus proche. Sa femme appelle le centre 15 à $7 \mathrm{~h} 37$, son époux venant de faire un malaise avec sueurs en allant aux toilettes, sans douleur thoracique associée. À l'interrogatoire du médecin régulateur, le

\footnotetext{
M. Dubocage $(\bowtie) \cdot H$. Delelis-Fanien $\cdot$ J. Guenezan Service des urgences adultes, SAMU 86, CHU Poitiers, 2 rue de la Milétrie, 86021 Poitiers, France

e-mail : mdubocag@gmail.com
}

Université de Poitiers, 15, rue de l'Hôtel Dieu, TSA 71117, 86073 Poitiers cedex 9, France patient se plaint d'une douleur thoracique rétrosternale non constrictive, apparue secondairement, sans irradiation, gênant l'inspiration profonde, jugée atypique, d'allure non coronarienne par le médecin régulateur. Le patient présente toujours de sueurs avec impossibilité de se mettre debout.

Le médecin régulateur prend alors la décision d'envoyer en première intention une ambulance privée à $7 \mathrm{~h} 42$. Cette dernière est équipée d'un dispositif permettant la télétransmission à la régulation des paramètres vitaux et d'un ECG 12 dérivations $\left(\right.$ Nomadeec $\left.^{\circledR}\right)$. Le premier ECG réalisé à $8 \mathrm{~h} 15$ par les ambulanciers met en évidence un syndrome coronarien aigu (SCA) avec sus-décalage du segment ST dans le territoire inférieur et un miroir dans le territoire antérieur (Fig. 2, panel A). Une équipe SMUR est déclenchée à $8 \mathrm{~h} 17$ pour se rendre au domicile du patient, réaliser un second ECG à 8 h40 (Fig. 2, panel B) et débuter les traitements médicamenteux appropriés. Le patient est transporté aux urgences cardiologiques du centre hospitalier universitaire. Il y est admis à 9 h1 18 où il bénéficie d'une coronarographie avec angioplastie, pose de deux stents actifs implantés sur l'artère coronaire droite et sur l'interventriculaire antérieure. Au total, le délai entre le début de la douleur et la revascularisation a été de moins de deux heures. Le patient est resté hospitalisé cinq jours en cardiologie, avant de retourner au domicile en attendant de se rendre dans un centre de rééducation.

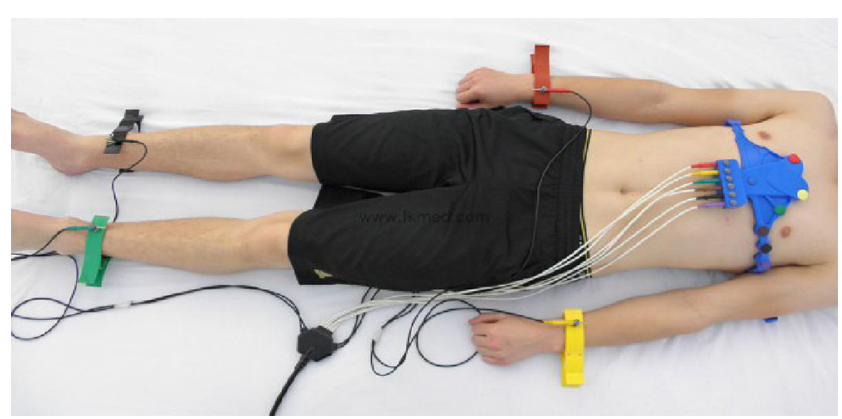

Fig. 1 Ceinture thoracique à électrocardiogramme (Nomadec ${ }^{\circledR}$, Exelus, Latresne, France) 


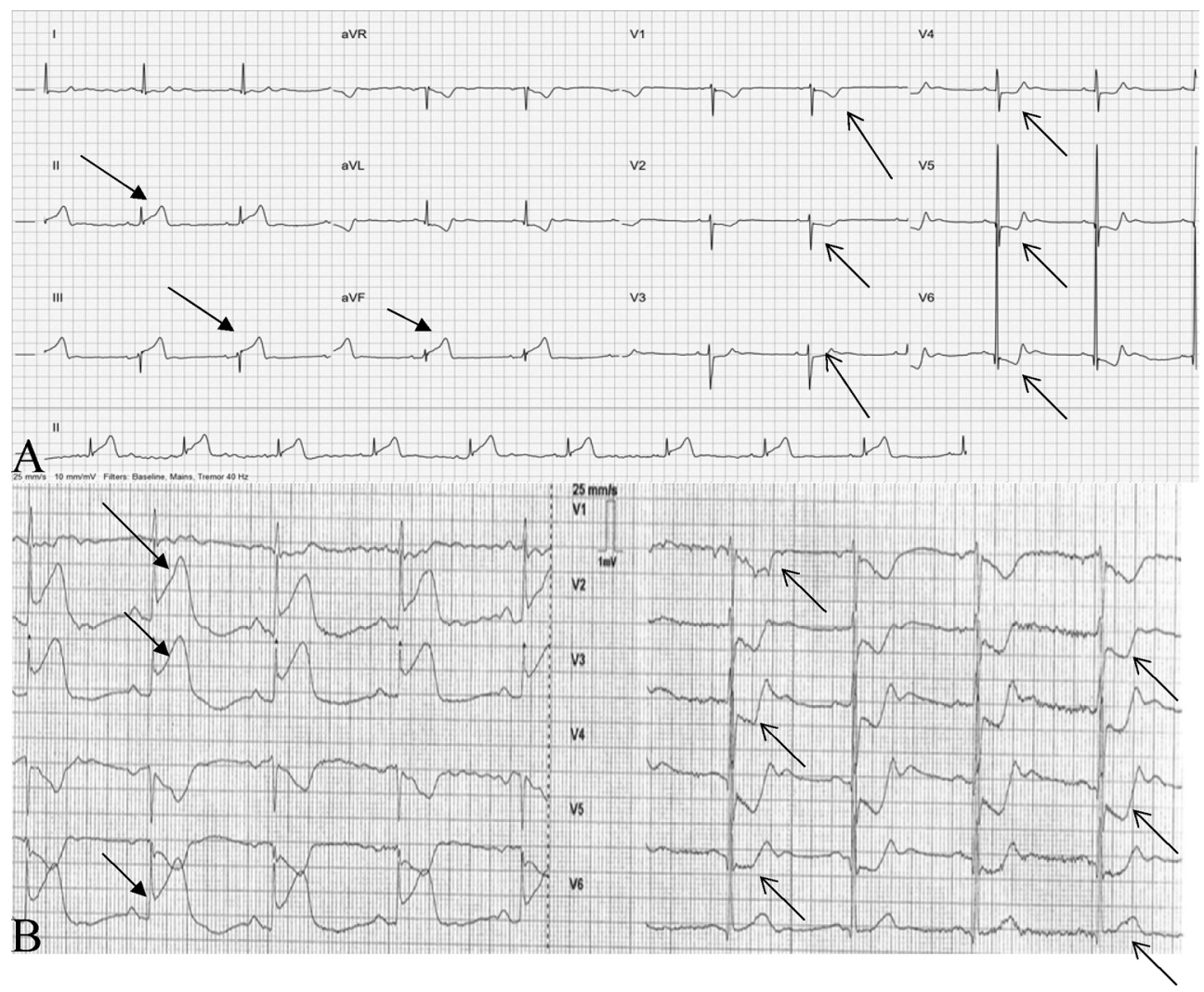

Fig. 2 Électrocardiogrammes. A : ECG réalisé à $8 \mathrm{~h} 15$ par le système Nomadeec ${ }^{\circledR}$ (Exelus, Latresne) ; B : ECG réalisé à 8 h40 par le SMUR montrant un sus-décalage dans le territoire inférieur (flèches à gauche) et un aspect de miroir en antérieur (flèches à droite)

\section{Discussion}

Ce cas clinique illustre l'intérêt potentiel de la réalisation d'un ECG par une équipe paramédicale et de sa télétransmission au Centre 15 dans le diagnostic préhospitalier d'un SCA en cas d'indisponibilité des équipes SMUR, d'isolement géographique des patients ou de décision initialement inappropriée de la part du médecin régulateur du Centre 15, comme dans le cas présenté ici. La formation des ambulanciers, équipés du système Nomadeec ${ }^{\circledR}$, est réalisée par la société qui commercialise ce dispositif sous la supervision d'un directeur médical. Cependant, l'envoi systématique d'une équipe SMUR en cas de suspicion de SCA, lorsqu'elle est disponible, reste bien sûr le seul moyen de garantir une prise en charge optimale dans les délais les plus courts.

Néanmoins, il est souvent difficile de faire la part des choses au moment de l'appel initial et une équipe SMUR est alors souvent déclenchée de manière systématique au domicile, compliquant la gestion des moyens médicaux souvent de moins en moins importants. De nombreux patients ayant un SCA présentent initialement une symptomatologie atypique telle que des vertiges ou encore des signes digestifs. A contrario, de nombreuses douleurs thoraciques ne sont pas d'origine cardiaque.
Notre cas est un exemple où la symptomatologie atypique présentée initialement par le patient cache une urgence réelle et prioritaire du préhospitalier qui méritait l'envoi d'un SMUR. Le fait que l'ambulance soit équipée d'un système de télétransmission, permettant la réalisation d'un ECG 12 dérivations, a permis de diminuer au maximum la perte de temps pour le patient en corrigeant une orientation diagnostique initiale inappropriée, d'orienter le patient dans un centre de soins adapté et de lui permettre de bénéficier d'une coronarographie avec angioplastie dans les délais actuellement recommandés.

De nombreuses études ont évalué l'intérêt de la télémédecine dans le suivi de pathologies chroniques, pour le monitorage de patients hospitalisés ou encore dans les urgences chirurgicales de la main [1-3]. D'autres ont évalué la gestion des accidents vasculaires cérébraux et l'intérêt de la fibrinolyse grâce à la télémédecine [4-6]. La télémédecine en préhospitalier est en plein développement. De plus en plus d'études évaluent sa faisabilité et l'intérêt de sa réalisation au sein des ambulances [6-9]. Elles ont montré l'intérêt des ECG télétransmis notamment sur la détection plus précoce de SCA de présentation atypique ou dans des milieux plus compliqués d'accès comme dans les centres pénitenciers [9].

Notre cas témoigne que la télémédecine apporte des informations supplémentaires au médecin régulateur pour 
optimiser la prise en charge des patients, notamment dans les cas de douleurs thoraciques atypiques, ou encore dans l'optimisation de la gestion des équipes de secours [10-12]. Ce système permet également de rattraper des erreurs de jugement, notamment dans des régulations difficiles, où l'expérience et la sensibilité du médecin décideur ne suffit malheureusement pas toujours à prendre la bonne décision. Son intérêt pourrait également être étendu à l'optimisation de la prise en charge des patients en milieu rural en raison de l'éloignement des moyens médicaux et du plateau technique. Ce dispositif offre donc des possibilités multiples, en termes de gestion des équipes, et permettrait aussi de débuter des thérapeutiques.

Nous rapportons un cas mais seule une étude prospective bien menée pourra confirmer le bénéfice d'un tel système sur le devenir des patients. De même, ce système ne doit en aucun cas se substituer à l'envoi d'une équipe SMUR, notamment lors d'une douleur thoracique évoquant fortement une origine coronarienne à l'appel. Enfin, l'usage de la télémédecine peut être pris en défaut dans certaines zones rurales où la couverture du réseau téléphonique mobile est insuffisante [13].

En conclusion, cet outil de télémédecine d'aide à la régulation offre un nouvel éventail de possibilités au médecin régulateur du Centre 15 sans pour autant remplacer une équipe SMUR ; il convient encore d'en réfléchir les usages et limites, d'en analyser les possibles dérives pour en faire un outil performant et efficient.

Liens d'intérêt : Les auteurs déclarent ne pas avoir de lien d'intérêt.

\section{Références}

1. Al Tout YA, Taleb C, Kosayer M, et al (2010) Télémédecine et urgences de la main: étude de faisabilité. In: Bustamante K,
Annales de chirurgie plastique esthétique. Elsevier Masson Ed., Paris, pp 8-13

2. Mackintosh N, Terblanche M, Maharaj R, et al (2016) Telemedicine with clinical decision support for critical care: a systematic review. Syst Rev 5:176

3. Flodgren G, Rachas A, Farmer AJ, et al (2015) Interactive telemedicine: effects on professional practice and health care outcomes. Cochrane Database of Syst Rev 2015;9:CD002098

4. Smith SNC, Govindarajan P, Padrick MM, et al (2016) A lowcost, tablet-based option for prehospital neurologic assessment The iTREAT Study. Neurology 87:19-26

5. Barrett KM, Pizzi MA, Kesari V, et al (2016) Ambulance-based assessment of NIH Stroke Scale with telemedicine: A feasibility pilot study. J Telemed Telecare 23:476-483

6. Amadi-Obi A, Gilligan P, Owens N, et al (2014) Telemedicine in pre-hospital care: a review of telemedicine applications in the pre-hospital environment. Int J Emerg Med 7:29

7. Yperzeele L, Van Hooff RJ, De Smedt A, et al (2014) Feasibility of Ambulance-Based Telemedicine (FACT) study: safety, feasibility and reliability of third generation in-ambulance telemedicine. PLoS One 9:e110043

8. Felzen M, Brokmann JC, Beckers SK, et al (2016) Improved technical performance of a multifunctional prehospital telemedicine system between the research phase and the routine use phase-an observational study. J Telemed Telecare 23:402-09

9. Bergrath S, Rossaint R, Lenssen N, et al (2013) Prehospital digital photography and automated image transmission in an emergency medical service-an ancillary retrospective analysis of a prospective controlled trial. Scand J Trauma Resusc Emerg Med $21: 3$

10. Bergrath S, Czaplik M, Rossaint R, et al (2013) Implementation phase of a multicentre prehospital telemedicine system to support paramedics: feasibility and possible limitations. Scand J Trauma Resusc Emerg Med 21:54

11. Munjal KG, Shastry S, Loo GT, et al (2016) Patient perspectives on EMS alternate destination models. Prehosp Emerg Care 20:705-11

12. Haskins PA, Ellis DG, Mayrose J (2002) Predicted utilization of emergency medical services telemedicine in decreasing ambulance transports. Prehosp Emerg Care 6:445-48

13. Cho SJ, Kwon IH, Jeong J (2015) Application of telemedicine system to prehospital medical control. Healthc Inform Res 21:196-200 